\title{
NCEIS-Buckets: A Novel Counter Estimation Information Structure that Relative Mistake for Network Measurement
}

\author{
Maddali M. V. M. Kumar, Grandhi Ushaswini \\ Assistant Professor, Dept. of MCA, St. Ann's College of Engineering \& Technology, Chirala. \\ PG Scholar, Dept. of MCA, St. Ann's College of Engineering \& Technology, Chirala.
}

\begin{abstract}
Estimation abilities are fundamental for an assortment of system applications, for example, stack adjusting, steering, reasonableness, and interruption recognition. These abilities require substantial counter clusters so as to screen the traffic of all system streams. While ware SRAM recollections are equipped for working at line speed, they are too little to even think about accommodating huge counter clusters. Past works proposed estimators, which exchange exactness for decreased space. In any case, so as to precisely gauge the biggest counter, these techniques bargain the exactness of the littler counters. In this paper, we present a shut frame portrayal of the ideal estimation work. We at that point present autonomous counter estimation basins, a novel calculation that enhances estimation precision for all counters. This is accomplished by isolating the streams to cans and designing the ideal estimation work as indicated by each can's counter scale. We demonstrate a more tightly upper bound on the relative mistake and exhibit a precision enhancement of up to multiple times on genuine Internet bundle follows.
\end{abstract}

Keywords: Communications innovation, correspondence frameworks, PC systems, PC organize the executives, arrange security.

\section{INTRODUCTION:}

A. Foundation:Counter exhibits are basic in system estimations and bookkeeping. Regularly, estimation applications track a few million streams [1] and their counters are refreshed with the landing of each bundle. These capacities are an essential empowering factor for systems administration calculations in numerous fields, for example, stack adjusting, steering, reasonableness, organize reserving and interruption discovery [3]. Kumar et al. [11] proposed is Dynamic Energy Efficient Distance Aware for the Energy Efficient Cluster selection mechanisms in the Wireless Sensor Networks. The primary principle is selection of cluster head is based on the principle of Residual Energy Distance Algorithms. The algorithm focusses on the selection of the Cluster head in the network based on the distance, RSSI and the new term called Rank of the Nodes. The low energy consumption has been achieved based on the distance and the signal strength. The Cluster head selection is based on the Residual Energy and Distance principles.Counter clusters are likewise utilized in prominent rough considering representations such multi organize channels [8] and tally min portray [9], just as in system checking designs. Such models are utilized to gather and dissect measurements from many systems administration gadgets [14]. Execution of counter exhibits is especially testing because of the prerequisite to work at line speed. Despite the fact that item SRAM recollections are quick enough for this errand, they don't meet the space necessities of present day counter clusters. Actualizing a counter exhibit totally in SRAM is along these lines over the top expensive [15]. Counter estimation calculations utilize shorter counters, e.g., 12-bits rather than 32bits, at the expense of a little mistake. Upon bundle entry, a counter is just increased with a specific likelihood that relies upon its present esteem. So as to keep the relative blunder uniform, little qualities are increased with high likelihood and huge ones with low likelihood. An emphestimation work is utilized so as to decide these probabilities and gauge the genuine estimation of a counter. Estimation capacities can be scaled to accomplish higher tallying limit at the expense of a bigger estimation blunder. Kumar at el.[18] introduce astructure for detecting and localizing performance irregularities in light of utilizing a dynamic test empowered measurement framework deployed on the periphery of a network organization. Boolean system tomography is an effective tool to infer the state (working/cancelled) of individual hubs from path level calculations extracted by edge-hubs. We think about the issue of optimizing the ability of recognizing system failures through the 


\section{International Journal of Research in Advent Technology, Vol.7, No.2, February 2019 E-ISSN: 2321-9637 \\ Available online at www.ijrat.org}

implementation of monitoring methods. Finding an ideal solution is NP-hard and an expansive group of work has been given to heuristic methodologies giving lower bounds. Dissimilar to past works, we give upper bounds on the highest number of identifiable hubs, given the number of monitoring paths and various constraints on the system topology, the routing methodology, what's more, the highest path length. The proposed upper bounds describes to a major limit on the identifiability of failures by means of Boolean system tomography. This investigation gives experiences on the most proficient method to design topologies and related monitoring schemes to accomplish the highest identifiability under different network settings.Existing counter estimation strategies experience the ill effects of the accompanying issue when confronting skewed outstanding tasks at hand, as is normal in PC organizes, a marvel known as overwhelming hitters. Pleasing the checking limit required by the overwhelming hitters powers utilizing an extensive estimation work scale. Be that as it may, since the substantial hitters frequently share a similar capacity scale as different counters, the estimation mistakes for little counters, which relate to most of things, turn out to be vast.

B. Commitments:In this work we present Novel Counter EstimationInformation StructureBuckets (NCEIS-Buckets), a novel counter estimation system that diminishes the general blunder by effectively using various counter scales. The fundamental rule of NCEIS-Buckets is delineated in Figure 1. In this model, the biggest counter (D) must be evaluated with an extensive scale and an overall mistake of $10 \%$. In the customary methodology, this mistake applies to all counters, as outlined in Figure 1(a). Figure 1(b) indicates what happens when the cluster is divided into free pails. Counter D is still evaluated with a blunder of $10 \%$, however for this situation the mistake applies just to counters inside a similar can. Alternate pails can utilize littler scales and appreciate bring down relative mistake. Thus, the general blunder is diminished. NCEIS-Buckets makes utilization of the ideal estimation work that was recently known just in recursive shape. We present an express portrayal and give an all-inclusive examination to this capacity. While the recursive portrayal required extra memory to keep up a precomputed exhibit of estimation esteems, the shut shape portrayal requires no such memory overhead. This permits NCEIS-Buckets to keep up numerous duplicates of the capacity with various scales without requiring more memory. Also, the shut shape portrayal empowers the formal examination incorporated into this paper.

A:12, B:98, C:1, D:10000, E:3, F:2, G:4, H:7, I:500, J:10, K:20, L:1

$10 \%$ error

(a)

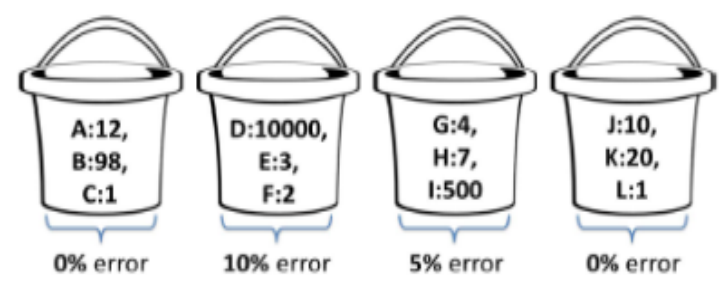

(b)

Fig. 1. An overview of NCEIS-Buckets vs. existing counter estimationapproaches.

C. Duties:In this work we present Novel Counter EstimationInformation Structure Buckets (NCEISBuckets), a novel counter estimation framework that lessens the general screw up by successfully utilizing different counter scales. The major standard of NCEIS-Buckets is outlined in Figure 1. In this model, the greatest counter (D) must be assessed with a broad scale and a general mix-up of $10 \%$. In the standard strategy, this oversight applies to all counters, as illustrated in Figure 1(a). Figure 1(b) demonstrates what happens when the group is partitioned into free buckets. Counter D is still assessed with a bungle of $10 \%$, anyway for this circumstance the error applies just to counters inside a comparable can. Substitute buckets can use humbler scales and value cut down relative mix-up. Subsequently, the general screw up is decreased. NCEIS-Buckets makes use of the perfect estimation work that was as of late known just fit as a fiddle. We present an express depiction and give a comprehensive examination to this limit. While the recursive depiction required additional memory to keep up a precomputed show of estimation regards, the close shape depiction requires no such memory overhead. This licenses NCEIS-Buckets to keep up various copies of the limit with different scales without requiring more memory.

\section{NCEIS-BUCKETS PARAMETER CHOICE}

We presently depict the way toward picking the parameters to limit the upper bound from Theorem 6 and afterward Theorem 7. In the standard situation, 


\section{International Journal of Research in Advent Technology, Vol.7, No.2, February 2019 E-ISSN: 2321-9637 \\ Available online at www.ijrat.org}

we have constrained space for our information structure of $\mathrm{T}$ bits. We for the most part have an upper destined for M, e.g., by duplicating the greatest bolstered traffic rate by the greatest estimation time. In the event that $M$ is as yet obscure, we can utilize the most extreme whole number we can speak to. $\mathrm{N}$ could likewise be given, as the greatest number of streams the systems administration gadget bolsters.

\section{CONCLUSION}

In this work, we have presented NCEIS-Buckets, a novel counter estimation information structure that limits the relative mistake. NCEIS-Buckets utilizes the ideal estimation work with a scale that is improved freely for each basin. We initially portrayed an unequivocal portrayal of this capacity, which was recently known just in recursive shape. We broadened its investigation and demonstrated a technique to gauge the impact of upscale activities on the relative blunder. This capacity is utilized in NCEIS-Buckets to limit the blunder in each container. NCEIS-Buckets is powerfully designed to adjust to the developing counters. For commonsense arrangements, it very well may be actualized without worldwide activities while giving comparative precision. We demonstrated an upper bound to NCEIS-Buckets' general relative mistake, which is essentially littler than that of past estimation calculations. Specifically, we exhibited a decrease of up to multiple times in this upper bound when connected to traffic attributes of genuine remaining tasks at hand. NCEIS-Buckets additionally accomplishes indistinguishable most extreme relative mistake from the ideal capacity. Furthermore, we broadly assessed NCEIS-Buckets with four Internet parcel follows and exhibited a decrease of up to multiple times in general blunder. NCEIS-Buckets accomplishes an enhancement in precision notwithstanding when it is given significantly less space than the choices. At last, we have demonstrated that NCEIS-Buckets is altogether more exact than the main choices for a wide scope of memory imperatives. In this work, we disclosed how to perform decrements and downscaling. supplanting the counters in the above with estimators could conceivably enhance their space to exactness proportion. Another promising part of the above is that estimators can restore the evaluated an incentive in $\mathrm{O}(1)$ time, subsequently keeping up the entrance effectiveness of such consolidated plans.

\section{REFERENCES}

[1] A. Metwally, D. Agrawal, and A. EI Abbadi, "Efficient computation of frequent and top-k elements in data streams," in Proc. 10th Int. Conf. Database Theory (ICDT), 2005, pp. 398412.

[2] R. M. Karp, S. Shenker, and C. H. Papadimitriou, "A simple algorithm for finding frequent elements in streams and bags," ACM Trans. Database Syst., vol. 28, no. 1, pp. 51-55, Mar. 2003.

[3] R. Ben-Basat, G. Einziger, R. Friedman, and Y. Kassner, "Heavy hitters in streams and sliding windows," in Proc. IEEE Conf. Comput. Commun. (INFOCOM), Apr. 2016, pp. 1-9.

[4] D. Ficara, A. Di Pietro, S. Giordano, G. Procissi, and F. Vitucci, "Enhancing counting bloom filters through Huffman-coded multilayer structures," IEEE/ACM Trans. Netw., vol. 18, no. 6, pp. 1977-1987, Dec. 2010.

[5] S. Cohen and Y. Matias, "Spectral bloom filters," in Proc. ACM SIGMOD, 2003, pp. 241-252.

[6] G. Einziger and R. Friedman, "Counting with TinyTable: Every bit counts!” in Proc. Int. Conf. Distrib. Comput. Netw. (ICDCN), 2016, p. 27.

[7] R. Morris, "Counting large numbers of events in small registers," Commun. ACM, vol. 21, no. 10, pp. 840-842, 1978.

[8] R. Stanojevic, "Small active counters," in Proc. IEEE INFOCOM, May 2007, pp. 2153-2161.

[9] C. Hu et al., "Discount counting for fast flow statistics on flow size and flow volume," IEEE/ACM Trans. Netw., vol. 22, no. 3, pp. 970-981, Jun. 2014.

[10] C. Hu and B. Liu, "Self-tuning the parameter of adaptive non-linear sampling method for flow statistics," in Proc. Int. Conf. Comput. Sci. Eng. (CSE), Aug. 2009, pp. 16-21.

[11] Maddali M.V.M. Kumar, Dr. AparnaChaparala, "Dynamic Energy Efficient Distance Aware Protocol for the Cluster Head Selection in the Wireless Sensor Networks", Recent Trends in Electronics Information \& Communication Technology (RTEICT) 2017 2nd IEEE International Conference on, pp. 147-150, 2017.

[12] E. Tsidon, I. Hanniel, and I. Keslassy, "Estimators also need shared values to grow together," in Proc. IEEE INFOCOM, Mar. 2012, pp. 1889-1897.

[13] L. Yang et al., "Case: Cache-assisted stretchable estimator for high speed per-flow measurement," in Proc. IEEE INFOCOM, Apr. 2016, pp. 1-9. 
[14] C. Hu et al., "Accurate and efficient traffic monitoring using adaptive non-linear sampling method," in Proc. IEEE INFOCOM, Apr. 2008, pp. 26-30.

[15] Wand Network Research Group. Waikato Internet Traffic Storage 2009- 01-06 00:00-23:30 UTC, ISPDSL-I. Accessed: Apr. 17, 2018. [Online]. http://wand.net.nz/wits/ispdsl/1/

[16] P. Hick. (2001). CAIDA Anonymized 2008 Internet Trace, Equinix- Chicago 2008-03-.19 19:00-20:00 UTC, Direction A. [Online]. Available:

http://www.caida.org/data/monitors/passiveequinix-chicago.xml

[17] T. Li, S. Chen, and Y. Ling, "Per-flow traffic measurement through randomized counter sharing," IEEE/ACM Trans. Netw., vol. 20, no. 5, pp. 1622-1634, Oct. 2012.

[18] Maddali M.V.M. Kumar, G. Navya, "A Probabilistic Path Measurement Approach for Identifying Node Issues in Network Topologies", International Journal of Scientific Research in Science and Technology; Vol. - 04; Issue - 05; pp. 29-35; DOI: 15.11/IJSRST1841313, 2018

[19] M. Chen and S. Chen, "Counter tree: A scalable counter architecture for per-flow traffic measurement," in Proc. IEEE Int. Conf. Netw. Protocols (ICNP), 2015, pp. 111-122.

[20] N. Hua, B. Lin, J. J. Xu, and H. C. Zhao, "BRICK: A novel exact active statistics counter architecture," in Proc. ACM/IEEE ANCS, 2008, pp. 89-98.

[21] B.-Y. Choi, J. Park, and Z.-L. Zhang, "Adaptive random sampling for load change detection," in Proc. ACM SIGMETRICS, 2002, pp. 272-273.

[22] Y. Zhang, S. Singh, S. Sen, N. Duffield, and C. Lund, "Online identification of hierarchical heavy hitters: Algorithms, evaluation, and applications," in Proc. ACM IMC, 2004, pp. 101-114.

[23] A. Metwally, D. Agrawal, and A. EI Abbadi, "Efficient computation of frequent and top-k elements in data streams," in Proc. 10th Int. Conf. Database Theory (ICDT), 2005, pp. 398412.

[24] R. M. Karp, S. Shenker, and C. H. Papadimitriou, "A simple algorithm for finding frequent elements in streams and bags," ACM Trans. Database Syst., vol. 28, no. 1, pp. 51-55, Mar. 2003.

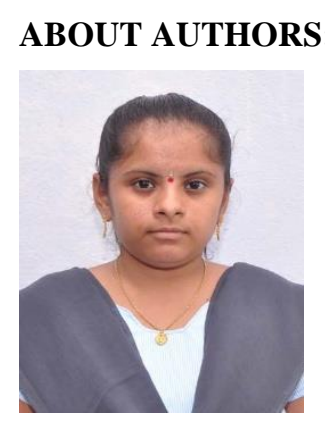

\section{Grandhi}

Ushaswiniis currently pursuing her MCA from Department of MCA, St. Ann's College Engineering and Technology, Chirala. She received her Bachelor of Science from ANU.

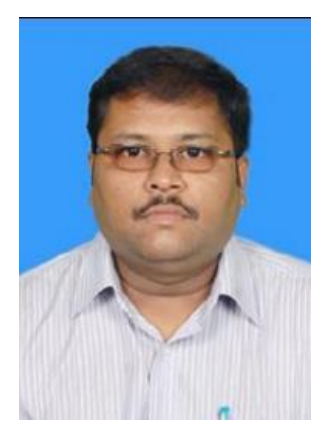

Mr. Maddali M. V. M. Kumar obtained his Master of Technology in CSE from JNT University Kakinada and currently pursuing his Ph.D. in Computer Science \& Engineering from Acharya Nagarjuna University, Guntur. $\mathrm{He}$ has published over five research and 20+ papers published in reputed International/National Journals and Conferences including Thomson Reuters (SCI \&WoS) and Conferences including IEEE, Springer and it is available online. $\mathrm{He}$ is a Life Member in CSI, IAENG and ISTE. His research work focuses on Computer Networks, Big Data \& Cloud Computing. 